\title{
ALCOOLEMIA E MORTES DE CAUSAS VIOLENTAS
}

\author{
BLOOD ALCOHOL CONCENTRATION AND VIOLENT DEATHS
}

\author{
Carolina M.C. de Paula', Maria A.M. Ruzzene², Bruno S. De Martinis ${ }^{3}$
}

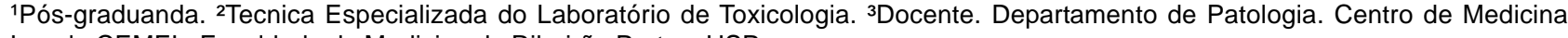
Legal - CEMEL. Faculdade de Medicina de Ribeirão Preto - USP.

CorRespondência: Prof. Dr. Bruno Spinosa De Martinis. CEMEL. Departamento de Patologia. Faculdade de Medicina de Ribeirão Preto -USP. Rua Tenente Catão Roxo, no 2418. 14051-140 Ribeirão Preto - SP, Brasil. Tel.: +55-16-36024424; fax: +55-16-36334476.

E-mail: cmcpaula@hotmail.com; martinis@fmrp.usp.br
\end{abstract}

Paula CMC, Ruzzene MAM, De Martinis BS. Alcoolemia e mortes de causas violentas. Medicina (Ribeirão Preto) $2008 ; 41$ (1): 24-9.

RESUMO: A Legislação Brasileira determina a realização do exame de alcoolemia apenas para vítimas fatais de acidentes de trânsito. Neste trabalho é discutida a necessidade da realização do exame toxicológico de álcool no sangue de todas as vítimas fatais de causa externa ou violenta, pois o uso abusivo do álcool pode estar intimamente relacionado com esses tipos de morte. O objetivo deste estudo foi analisar e conhecer a relação entre a concentração de álcool no sangue e as mortes de causa violenta, considerando os parâmetros sexo e faixa etária. Foram analisadas amostras de sangue de vítimas fatais de causa violenta provenientes de necropsia realizadas pelo Núcleo de Perícia Médico Legal de Ribeirão Preto (NPML-RP) no Centro de Medicina Legal da Faculdade de Medicina de Ribeirão Preta da Universidade de São Paulo, no período de 2002 a 2004. As análises de etanol foram realizadas pela técnica de cromatografia em fase gasosa com detector de ionização por chama e Headspace. Os resultados mostraram que nos acidentes, em especial nos acidentes de trânsito, a maior parte dos indivíduos envolvidos apresentava alcoolemia positiva, seguido dos homicídios e suicídios. Houve predominância das amostras do sexo masculino, sendo que mais que $50 \%$ das vítimas apresentavam alcoolemia positiva, com valores acima do permitido pelo Código de Trânsito Brasileiro, que é de 0,6 g/l. Os resultados sugerem que o álcool está altamente correlacionado com as mortes de causas violentas, principalmente nos acidentes de trânsito e envolvendo indivíduos jovens do sexo masculino.

Descritores: Álcool. Morte violenta. Toxicologia Forense. Cromatografia em fase gasosa.

\section{1- INTRODUÇÃO}

Relato da Organização Mundial de Saúde ${ }^{1}$ indicaram que aproximadamente dois bilhões de pessoas no mundo consomem bebidas alcoólicas. Este consumo pode ter provocado 1,8 milhões de mortes, sendo $1 / 3$ delas provocada por traumas. Estima-se que aproximadamente $20-30 \%$ destas mortes têm causas externas, como homicídios e acidentes de trânsito.

No Brasil, as causas externas foram responsáveis por 128.790 óbitos no ano de 2003, corresponden- do à terceira causa de óbito ${ }^{2}$. O consumo de álcool aumenta o fator de risco de morte violenta ${ }^{3}$. Assim, a investigação da presença de álcool em amostras coletadas de vítimas de morte violenta pode ser de grande relevância na elucidação de uma morte ocorrida nestas circunstâncias.

Pesquisas têm demonstrado que a prevalência de alcoolemia positiva em vítimas não fatais de diferentes causas externas é de $6 \%$ a $34 \%$, e que nos casos fatais a prevalência do álcool nas amostras de sangue é ainda maior ${ }^{4}$. As causas externas constituem a ter- 
ceira causa de óbito na população geral do Brasil, colocando-se como a primeira causa de óbito na faixa etária de cinco a 40 anos, nos Estados Unidos, representam a quarta causa de óbito na população geral ${ }^{4}$.

Atualmente, está bem evidenciado que o álcool é a droga mais abusada em todo o mundo, e vários trabalhos têm demonstrado a estreita relação entre o abuso de álcool e violência ${ }^{3,5,6}$. O objetivo do presente trabalho é descrever a prevalência e a concentração de álcool no sangue de vítimas fatais de causa violenta, e sua interação quanto à idade e gênero, dentre todos os corpos necropsiados no CEMEL/FMRP/USP.

\section{2- MATERIAL E MÉTODOS}

\section{1- Coleta de dados}

Cabe salientar que, até 1998, o acesso às informações sobre mortes violentas com finalidade de pesquisa científica era bastante limitado, pois os serviços médico-legais eram desvinculados das universidades. Entretanto, um acordo entre o NPML-RP subordinado à Secretaria de Segurança Pública do Estado de São Paulo (SSP-SP) e a Faculdade de Medicina de Ribeirão Preto, permitiu a criação do Centro de Medicina Legal. Tal acordo, firmado em 1996, prevê a "cooperação técnico-científica mútua nas áreas de perícia e pesquisa no campo da Medicina Legal, objetivando a celeridade e o aperfeiçoamento das investigações criminais". Este modelo de prática médicolegal é o primeiro desenvolvido no País desde o fim do regime militar ocorrido no Brasil até $1985^{7}$.

Por meio deste acordo, a pesquisa foi desenvolvida no Laboratório de Toxicologia Forense do CEMEL/FMRP/USP. Para a realização do projeto, foram utilizados dados referentes às necropsias realizadas pelo NPML-RP, e dos boletins de ocorrência policiais arquivados neste Núcleo, de 2002 a 2004.

Neste intervalo de tempo, das 2072 necropsias de vítimas de morte violenta, 400 amostras de sangue foram submetidas ao laboratório para a realização do exame de alcoolemia.

Com relação às causa jurídicas de morte, as amostras foram classificadas como provenientes de vítimas fatais de acidentes, sendo a maioria acidentes de trânsito, homicídios, suicídios e outras causas de morte. Os dados utilizados para o estudo das causas jurídicas da morte violenta foram obtidos dos laudos necroscópicos. Como critério de inclusão, foi considerado as amostras obtidas de indivíduos com idade acima de 10 anos.

\section{2-Análise de amostras de sangue post-mortem}

As amostras de sangue foram coletadas durante as necropsias e foram acondicionadas em frascos contendo fluoreto de sódio como conservante, para evitar o crescimento de bactérias que transformam a glicose presente no sangue em etanol, resultando em falsos positivos.

As amostras foram analisadas por cromatografia em fase gasosa com detector de ionização por chama (GC-FID) (Varian 3380®), utilizando a técnica de Headspace para a introdução da amostra no cromatógrafo ${ }^{8}$.

\section{3- Análise estatística}

Para a detecção de diferenças estatísticas entre os diferentes parâmetros estudados, foi utilizado o teste de qui-quadrado com nível de significância de $1 \%(p<0,01)$. Nas análises estatísticas foi utilizado o pacote estatístico SAS ${ }^{\circledR}$ (1995).

\section{3- RESULTADOS E DISCUSSÃO}

Foram consideradas positivas as amostras com alcoolemia igual ou superior a $0,01 \mathrm{~g}$ de álcool por litro de sangue. Como o Código de Trânsito Brasileiro estabelece o valor máximo permitido de $0,6 \mathrm{~g} / \mathrm{l}$ de sangue para a condução de veículos, os casos positivos foram divididos em duas categorias: (a) concentração de etanol menor que $0,6 \mathrm{~g} / \mathrm{l}$ e maior que $0,01 \mathrm{~g} / \mathrm{l}$; (b) concentração igual ou superior a $0,6 \mathrm{~g} / \mathrm{l}$.

Considerando as amostras recebidas para a determinação de alcoolemia, 75,7\% (317 amostras) foram de amostras provenientes de vítimas de acidentes, sendo que $86,8 \%$ ( 277 amostras) foram vítimas de acidentes de trânsito. 10\% (40 amostras) foram de vítimas de homicídios, 8,2\% (33 amostras) foram vítimas de suicídios e 8,2\% (33 amostras) foram amostras provenientes vítimas de morte de causa externa, porém de maneira não esclarecida. Do total de amostras recebidas para análise toxicológica, 23 amostras $(5,4 \%)$ não apresentavam informação sobre a idade e, apesar de terem sido analisadas, não foram consideradas neste trabalho. Assim, para a apresentação dos resultados foram considerados 400 casos que apresentavam todas as informações sobre as variáveis consideradas neste estudo.

Foi observado que, de 400 amostras de sangue analisadas, 47,3\% (189 amostras) apresentaram alcoolemia negativa e 52,7\% (211 amostras) apresentaram resultados positivos. Destas, 58,3\% (123 amostras) 
apresentaram valores iguais ou superiores a $0,6 \mathrm{~g} / \mathrm{l}$, ou seja, acima do limite permitido pelo Código de Trânsito Brasileiro e 41,7\% (88 amostras) apresentaram resultados positivos, porém, abaixo do limite legal.

A Figura 1 mostra a distribuição da alcoolemia segundo a causa jurídica da morte violenta relacionada com a alcoolemia (negativa, positiva abaixo do limite máximo permitido e positiva acima do limite máximo permitido).

$\mathrm{O}$ teste do qui-quadrado mostrou que as proporções entre o número de amostras com resultados negativos e o número de amostras com resultados positivos podem ser considerados iguais $(50 \%$ para cada), indicando que não há prevalência de resultados positivos ou negativos. Porém, se considerarmos que em $50 \%$ das mortes existe o fator álcool envolvido, o cenário passa a ser preocupante. Avaliando apenas os casos positivos, observamos que o teste do quiquadrado indicou uma maior proporção dos casos com alcoolemia igual ou superior a $0,6 \mathrm{~g} / \mathrm{l}$.

\section{1- Alcoolemia positiva e sexo}

Observou-se predominância acentuada do sexo masculino nas análises de alcoolemia positiva, com
90,5\% dos casos (191 amostras). O teste do qui-quadrado mostrou que existe uma diferença significativa entre o número de indivíduos do sexo masculino e do sexo feminino para os casos positivos.

\section{2- Alcoolemia positiva, faixa etária e causa ju- rídica de morte}

A média de idade dos indivíduos que apresentaram alcoolemia positiva foi de 36,3 anos. Os acidentes de trânsito apresentaram uma média de 36,6 anos, os homicídios 32,3 anos, os suicídios 35,5 anos e as causas de morte violenta, porém indeterminada 36,7 anos.

Os indivíduos do sexo masculino na faixa etária de 35 a 44 anos estavam mais envolvidos em acidentes em geral, enquanto que na faixa etária de 18 a 34 anos houve prevalência de homicídios. Os suicídios ocorreram predominantemente em indivíduos na faixa etária de 18 a 24 anos.

Com relação aos indivíduos do sexo feminino, foi observado que a faixa etária mais envolvida com acidentes foi de 25 a 34 anos. Em relação às demais causas jurídicas de morte violenta, não foi possível inferir conclusões mais detalhadas devido ao pequeno número de mulheres envolvidas nesses tipos de mortes.

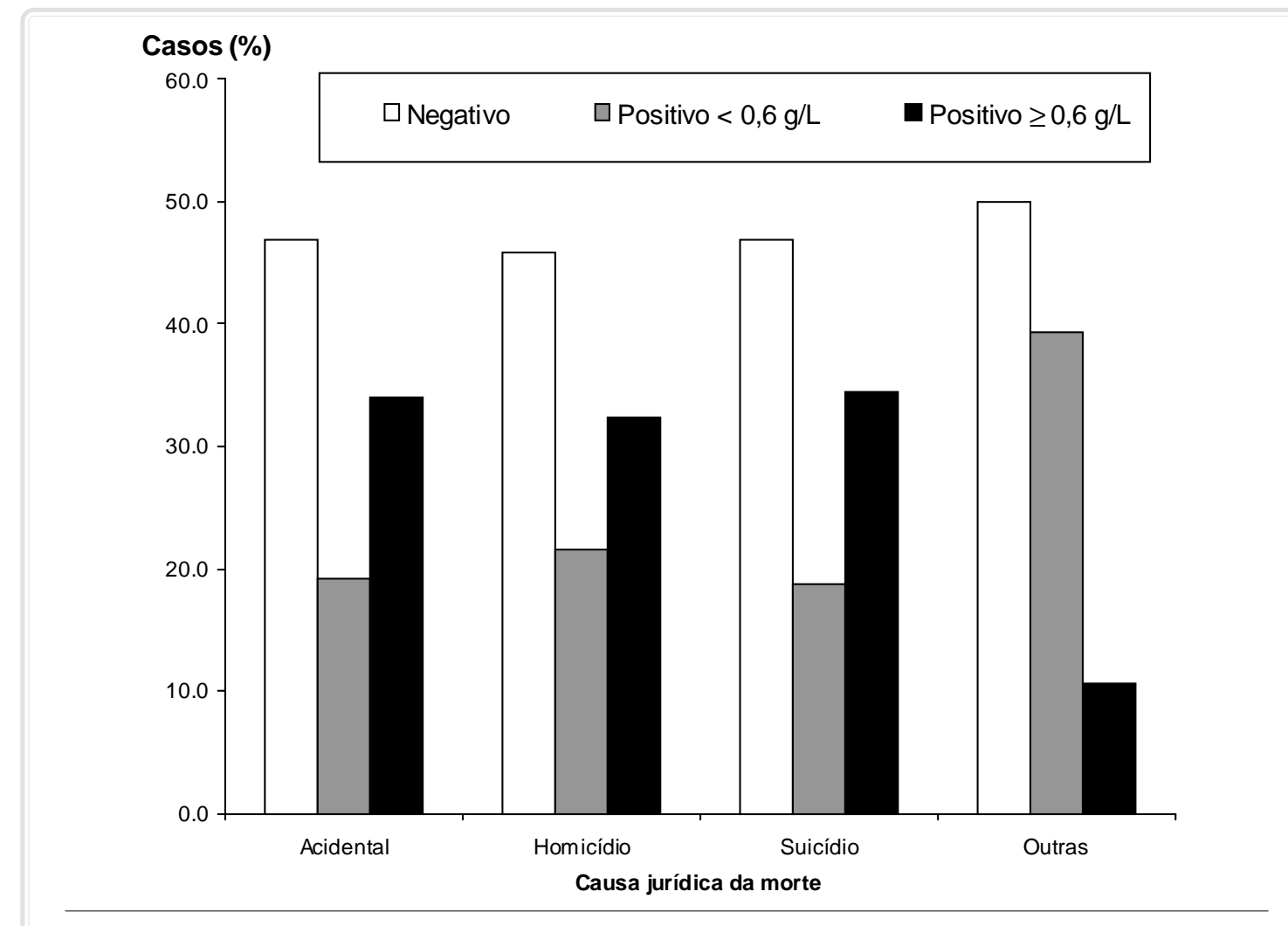

Figura 1: Distribuição da alcoolemia segundo a causa jurídica da morte. 


\section{3- Alcoolemia positiva, gênero e causa jurídica de morte violenta}

\section{Acidentes}

Considerando os acidentes em geral, 61,0\% das vítimas (161 casos) estavam alcoolizadas. Houve predominância de 93,4\% do sexo masculino (127 casos), que é estatisticamente significativo quando comparado com o número de mulheres.

Particularizando os acidentes de trânsito, foram analisadas 263 amostras, que representam $(86,8 \%)$ de todas as amostras analisadas, considerando todas as outras classificações de causa de morte violenta. Em $52 \%$ destas amostras (137 casos) a alcoolemia foi positiva $(<0,6 \mathrm{e}=0,6 \mathrm{~g} / \mathrm{l})$.

As análises estatísticas indicaram uma tendência de maior número de indivíduos com idade entre 18 e 24 anos sem separação entre homens ou mulheres, porém, no sexo masculino houve predomínio de jovens na faixa etária entre 20 e 29 anos, enquanto nas mulheres, a faixa etária mais encontrada foi entre 30 e 39 anos.

\section{Homicídios}

Considerando as análises realizadas em amostras de sangue de vítimas de homicídios, 54\% apresentavam alcoolemia positiva, sendo que $85 \%$ eram do sexo masculino. Do total de 40 homicídios, $47,5 \%$ (19 casos) foram causados por lesão de projétil de arma de fogo, 32,5\% (13 casos) vítimas de golpes de arma branca e $20 \%$ ( 8 casos) por homicídios causados por outros instrumentos.

As vítimas de homicídios por lesão de projétil de arma de fogo, na sua maioria, eram do sexo masculino, correspondendo a 89,5\% (17 casos), sendo que, $64.7 \%$ estavam alcoolizados.

As mortes por golpes de arma branca representaram 32,5\% (13 casos) dos homicídios, sendo que $92 \%$ ( 11 casos) eram do sexo masculino, e $61 \%$ destes apresentaram alcoolemia positiva.

\section{Suicídio}

As análises realizadas para os casos de suicídio mostraram alcoolemia positiva em 54\% (18 casos) das vítimas, sendo 94,1\% (17 casos) do sexo masculino. Entre as mortes por suicídio, a asfixia mecânica (enforcamento) foi responsável por 39,3\% (13 casos), a precipitação por $18 \%$ (6 casos) e $42,4 \%$ (14 casos) ocorreram por outros métodos.

Para a asfixia mecânica, $84,6 \%$ das amostras eram provenientes de indivíduos do sexo masculino e foi constatada alcoolemia positiva em $54,5 \%$.
Nas precipitações, $83 \%$ (4 casos) dos indivíduos eram do sexo masculino e $60 \%$ ( 2 casos) apresentaram alcoolemia positiva. Todas as mulheres que cometeram suicídio por precipitação estavam sob efeito do álcool.

\section{4- Causas jurídicas de morte violenta porém indeterminadas}

A maior parte destas amostras foi de indivíduos do sexo masculino (81\%, 24 casos), sendo que, do total das análises, 54,5\% (18 casos) apresentavam alcoolemia positiva.

No presente trabalho, a população estudada caracterizou-se, predominantemente, pelo sexo masculino, com proporção de 9:1. Embora a violência esteja fortemente vinculada ao consumo de bebidas alcoólicas ${ }^{5,6,9}$, esta associação varia, consideravelmente, com a quantidade ingerida ${ }^{10,11,12}$. Os efeitos do álcool contribuem com a probabilidade aumentada do comportamento agressivo, pois este afeta as funções cognitivas ${ }^{13}$ levando o individuo ao conflito, prejudicando sua habilidade emocional ${ }^{14}$. Estes comportamentos são mais predominantes em homens, uma vez que, quando alcoolizados, tendem a demonstrar suas características de dominação $0^{9,15}$.

Considerando as análises que resultaram positivas, $58 \%$ apresentaram valores acima de $0,6 \mathrm{~g} / \mathrm{l}$ (limite estabelecido pelo Código de Trânsito Brasileiro). Porém, mesmo sendo obrigatório a solicitação do exame de alcoolemia nas mortes por acidentes de trânsito, em menos da metade dos exames necroscópicos realizados pelo NPML-RP foram coletadas amostras de sangue para a realização das análises. Uma possível justificativa para esta defasagem é a dificuldade em promover a mudança de comportamento e postura dos profissionais que atuam em sala de necrópsia no sentido de perceberem a importância das análises toxicológicas como exame complementar para a elucidação dos casos e contribuição para a preparação dos laudos necroscópicos. Outra explicação é que, quando as vítimas inicialmente sobrevivem aos traumas e passam por procedimentos e tratamentos hospitalares, ficando internadas por horas ou dias, quando morrem e são encaminhadas para exame necroscópico, não há mais a presença do álcool devido à metabolização e eliminação do mesmo do organismo. Esta metabolização pode ser contabilizada através de cálculos retrospectivos e extrapolação da concentração de etanol quando se tem as informações de tempo decorrido entre o fato e o óbito, mas, já representa uma quantificação indireta de difícil intrepretação devido a grande variabilidade entre indivíduos. 
Em alguns casos, por falta de informações nos boletins de ocorrências encaminhados pela polícia juntamente com o corpo, as vítimas de acidentes de trânsito não puderam ser discriminadas entre pedestres, ciclistas, passageiros ou motoristas, dessa forma, a única comparação que pode ser feita com relação aos resultados apresentados pela literatura, diz respeito à porcentagem de positividade. Um estudo realizado na cidade de Ljubljana (Eslovênia) detectou que o álcool está presente em $76,3 \%$ das mortes de causa violen$\mathrm{ta}^{3}$. Outro estudo realizado em Budapeste (Hungria), demonstrou alcoolemia positiva em $66 \%$ das vítimas de acidentes de trânsito, sendo que, em $42 \%$ o valor máximo permitido por lei havia sido ultrapassado ${ }^{16}$. No nosso estudo, $52,7 \%$ dos casos avaliados considerando todas as causas de morte violenta, apresentavam alcoolemia positiva, sendo que, considerando os acidentes de trânsito, $53 \%$ das vítimas apresentavam positividade para álcool.

\section{4- CONCLUSÕES}

Os resultados obtidos neste trabalho confirmam que, o álcool é um fator importante e está altamente relacionado com as mortes de causas violentas apontando uma necessidade de leis e sanções mais rígidas.

Comparando os níveis de alcoolemia a grande maioria dos casos foram vítimas fatais de acidentes, principalmente os de trânsito, seguido dos homicídios causados por projétil de arma de fogo e arma branca e dos suicídios provocados por asfixia mecânica e precipitação.

A alta prevalência de utilização do álcool entre as vítimas de morte violenta sugere que outros estudos devem ser realizados para avaliar de forma mais definida o papel do álcool na potencialização das causas de mortes externas. A coleta de amostras para a determinação de alcoolemia em todas as mortes de causa violenta está longe de ser uma rotina para NPML-RP, e foi realizada apenas durante o período do trabalho em questão. Como os resultados obtidos indicam que existe uma relação entre o consumo de álcool e as mortes de causa violenta, podemos concluir que existe a necessidade de investigação em todas as causas jurídicas de morte violenta como resultado complementar ao exame necroscópico e para corroborar na elucidação da causa da morte. Tais informações podem contribuir para o estabelecimento de medidas legais, preventivas e punitivas com o objetivo de restringir o consumo abusivo do álcool pela população.

\section{5- AGRADECIMENTOS}

A CAPES pela bolsa de pós-graduação e a FAPESP pelo auxílio financeiro da pesquisa.

Paula CMC, Ruzzene MAM, De Martinis BS. Blood alcohol concentration and violent deaths. Medicina (Ribeirão Preto) 2008; 41 (1): 24-9.

ABSTRACT: Brazilian Legislation requests blood alcohol tests only for fatal victims of traffic accidents. In this work we will discuss the need of doing blood alcohol tests for all victims of violent death since abusive use of alcohol represents an important risk factor for all kinds of violent death. The proposal of this study was to analyze the relationship between blood alcohol concentration and the causes of violent death according to gender and age. Blood samples of fatal victims due to violence, from necroscopic examination performed at the Núcleo de Perícias Médico Legal de Ribeirão Preto (NPML-RP) at the Centro de Medicina Legal da Faculdade de Medicina de Ribeirão Preto da Universidade de São Paulo (CEMEL/FMRP/USP) were analyzed in the period from 2002 to 2004 . The ethanol analyses were done by gas chromatography using flame ionization detector and using Headspace technique for samples injection. The results showed that higher blood alcohol positivity was observed for victims of accidents, especially for traffic accidents, following by homicides, suicides and undetermined manner of violent deaths. It was observed also that samples collected from male victims were predominant and more than $50 \%$ of them presented blood alcohol above the limit established by the Brazilian traffic law which is $0.6 \mathrm{~g} / \mathrm{l}$. Results suggests that alcohol abused is highly correlated with violent deaths, mainly traffic accidents involving young males.

Keywords: Alcohol. Violent death. Forensic toxicology. Gas Chromatography. 


\section{REFERÊNCIAS}

1 - The World Health Report 2002: reducing risks, promoting healthy life. Geneva: WHO, 2002. 239p. Disponível em: em: http://www.who.int/whr/2002/en/ - Acesso em: 25 de setembro de 2005.

2 - Biblioteca Virtual em Saúde. Ministério da Saúde (BVSMS). Disponível em: http://bvsms.saude.gov.br/bvs/publicacoes/ saude_brasil_2005parte3.pdf - Acesso em: 02 de agosto de 2007.

3 - Skibin L, Bilban M, Balazic J. Harmful alcohol use of those who died a violent death (the extended region of Ljubljana 1995-1999). Forensic Sci Int 2005; 147: 49-52.

4 - Gazal-Carvalho C, Carlini-Cotrim B, Silva OA, Sauaia N. Blood alcohol content prevalence among trauma patients seen at a level 1 trauma center. Rev Saúde Pública 2002; 36(1):47-54.

5 - Pillon SC, O'Brien B, Piedra Chavez KA. The relationship between drugs use and risk behaviors in Brazilian university students. Rev Lat Am Enfermagem 2005, 13(2), 1169-1176.

6 - Zilberman ML, Blume SB. Domestic violence, alcohol and substance abuse. Rev Bras Psiquiatr 2005, Suppl 2:S51-5.

7 - Guimarães MA. The challenge of identifying deceased individuals in Brazil: from dictatorship to DNA analysis. Sci Justice 2003; 43(4):215-7.

8 - De Martinis BS, de Paula CM, Braga A, Moreira HT, Martin CC. Alcohol distribution in different postmortem body fluids. Hum Exp Toxicol. 2006; 25 (2): 93-7.
9 - Graham J. Policing and the mentally disordered. Scott Med J 2001; 46 (2): 38-9.

10 - Murdoch D, Pihl RO, Ross D. Alcohol and crimes of violence: present issues. Int J Addict 1990; 25 (9): 1065-81.

11 - Room R. Intoxication and bad behaviour: understanding cultural differences in the link. Soc Sci Med 2001; 53 (2): 189-98.

12 - Rossow I. Alcohol and homicide: a cross-cultural comparison of the relationship in 14 European countries. Addiction 2001; 96 (suppl 1): 77-92.

13 - Peterson JB, Pihl RO. Information processing, neuropsychological function, and the inherited predisposition to alcoholism. Neuropsychol Rev 1990; 1(4): 343-69.

14 - Pihl RO, Peterson J. Drugs and aggression: correlations, crime and human manipulative studies and some proposed mechanisms. J Psychiatry Neurosci 1995; 20 (2): 141-9.

15 - Mcclelland DC. Proceedings: drinking as a response to power needs in man. Psychopharmacol Bull 1974; 10 (4): 5-6.

16 - Toro K, Hubay M, Sotonyi P, Keller E. Fatal traffic injuries among pedestrians, bicyclists and motor vehicle occupants. Forensic Sci Int 2005; 151(2/3): 151-6.

Recebido para publicação em 27/08/2007

Aprovado para publicação em 13/05/2008 